\title{
molecules
}

ISSN 1420-3049

www.mdpi.com/journal/molecules

Article

\section{Pheonolic Compounds from the Fruits of Viburnum sargentii Koehne}

\author{
Yang Xie, Jing Wang, Yan-Mei Geng, Zhi Zhang, Yan-Fei Qu and Guang-Shu Wang * \\ School of Pharmaceutical Sciences, Jilin University, Changchun 130021, China; \\ E-Mails: 13514486691@163.com (Y.X.); 13578752256@163.com (J.W.); \\ gengym0102@163.com (Y.-M.G.); zz4548209@163.com (Z.Z.); wdcq2511@163.com (Y.-F.Q.) \\ * Author to whom correspondence should be addressed; E-Mail: wgs@jlu.edu.cn; \\ Tel.: +86-431-8561-9706.
}

Academic Editor: Derek J. McPhee

Received: 10 June 2015 / Accepted: 3 August 2015 / Published: 6 August 2015

\begin{abstract}
Seven phenolic compounds were isolated from the fruits of Viburnum sargentii Koehne by silica gel column chromatography and preparative HPLC. On the grounds of chemical and spectroscopic methods, their structures were identified as (-)-Epicatechin (1), 5,7,4'-trihydroxy-flavonoid-8-C- $\beta$-D-glucopyranoside (2), 1-(4-hydroxy-3-methoxyphenyl)2-[4-(3- $\alpha$-L-rhamnopyranoxypropyl)-2-methoxyphenoxy]-1,3-propane-diol (erythro) (3), 1-(4-hydroxy-3-methoxyphenyl)-2-[4-(3- $\alpha$-L-rhamnopyranoxypropyl)-2-methoxyphenoxy]1,3-propanediol (threo) (4), $(R)$-4-hydroxylphenol $O$-(6-O-oleuropeoyl)- $\beta$-D-glucopyranoside (5), (R)-3-methoxy-4-hydroxylphenol $O$-(6- $O$-oleuropeoyl)- $\beta$-D-glucopyranoside $\quad(6)$, quercetin-3-O-rutinoside (7). Compounds 5 and $\mathbf{6}$ are new monoterpene phenolic glycosides, compounds 1, 3 and 4 were isolated from the Viburnum genus for the first time, and compounds $\mathbf{2}$ and 7 from the Viburnum sargentii Koehne for the first time. Compounds 1-7 were also assayed for their antioxidant activities with DPPH free radicals.
\end{abstract}

Keywords: Viburnum sargentii; phenolic glycoside; monoterpene; epicatechin; quercetin

\section{Introduction}

Viburnum sargentii koehne, a deciduous shrub of Caprifoliaceae, is widely distributed in the northeast and northwest regions of China. As traditional Chinese medicines, the branches are used for 
rheumatoid arthritis and traumatic injuries, the leaves for boils, ringworm and skin itching, and the fruits for cough [1]. Although there are a number of the pharmacological studies of the fruits of Viburnum sargentii [2,3], the isolation and structure identification have not been investigated in detail. As one part of our Caprifoliaceae studies, the isolation and structure identification of chemical constituents from the fruits of Viburnum sargentii Koehne have been carried out, and we report the isolation and identification of two new monoterpene phenolic glycosides (5 and $\mathbf{6})$, together with five phenolic compounds 1, 2, 3, 4 and 7 in the present study (Figure 1).<smiles>Oc1cc(O)c2c(c1)O[C@H](c1ccc(O)c(O)c1)[C@H](O)C2</smiles>

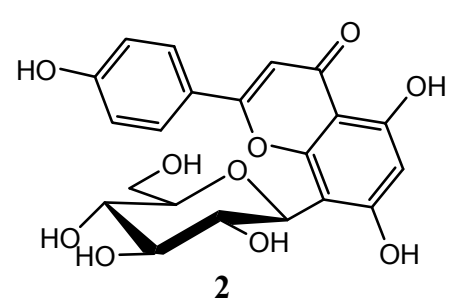

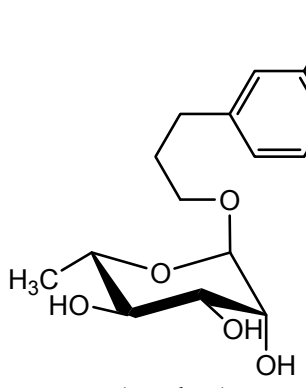

3 (erythro) 4 (threo)

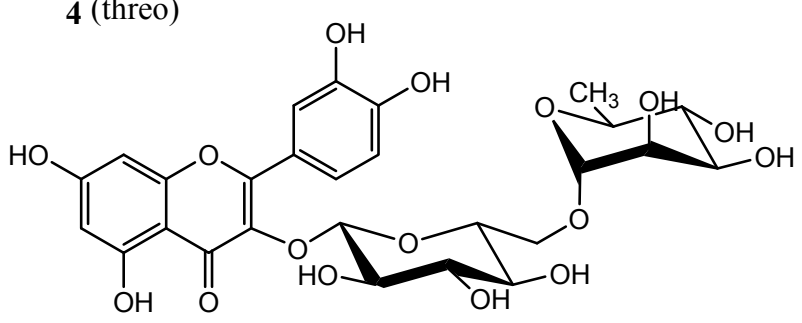

7

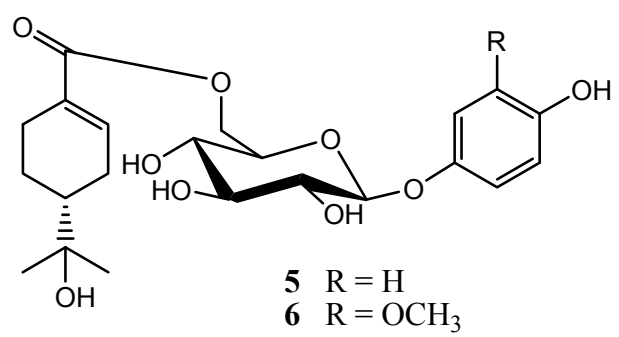

$\mathrm{O}-\mathrm{OCH}_{3}$<smiles>CC(C)(C)O</smiles>

$5 \mathbf{a}$

Figure 1. Chemical structures of compounds 1-7.

\section{Results and Discussion}

Compound 5 was obtained as colorless needles. Its HRESIMS displayed a $[\mathrm{M}+\mathrm{H}]^{+}$ion peak at $m / z$ 439.1957 (calcd for $\mathrm{C}_{22} \mathrm{H}_{31} \mathrm{O}$, 439.1968 ), indicating the molecular formula $\mathrm{C}_{22} \mathrm{H}_{30} \mathrm{O}_{9}$. Acidic hydrolysis of 5 yielded D-glucose as a sugar residue. The ${ }^{1} \mathrm{H}$ - and ${ }^{13} \mathrm{C}-\mathrm{NMR}$ spectra showed two $2 \mathrm{H}$ doublets $\left(\delta_{\mathrm{H}} 6.83(2 \mathrm{H}, \mathrm{d}, J=8.7 \mathrm{~Hz}), 7.03(2 \mathrm{H}, \mathrm{d}, J=8.7 \mathrm{~Hz})\right)$ and signals of six symmetric aromatic $\mathrm{C}$-atoms at $\delta_{\mathrm{C}} 154.3(\mathrm{C}), 152.1(\mathrm{C}), 119.5(2 \mathrm{CH})$, and $117.4(2 \mathrm{CH})$, arising from a symmetrically 1,4-O-disubstituted benzene ring moiety, a set of signals characteristic of $\beta$-D-glucopyranosyl moiety (anomeric $\mathrm{H}$-atom signal at $\delta_{\mathrm{H}} 4.88(\mathrm{~d}, J=7.5 \mathrm{~Hz})$ ). The ${ }^{13} \mathrm{C}-\mathrm{NMR}$ and DEPT spectra showed ten carbon signals comprising one carboxylic $\left(\delta_{\mathrm{C}} 168.2\right)$, one trisubstituted double bond $\left(\delta_{\mathrm{C}} 142.2,131.5\right)$, two methyl ( $\left.\delta_{C} 27.1,26.6\right)$, three methylene $\left(\delta_{c} 29.1,26.9,24.9\right)$, one methine $\left(\delta_{c} 45.7\right)$, and one oxygen-bearing quaternary carbon $\left(\delta_{c} 72.2\right)$. These observations suggested the presence of an oleuropeic acid unit [4,5]. The positions of the oleuropeoyl ester and glycosidic linkages in $\mathbf{5}$ were 
established by 2D-NMR experiments. In the HMBC spectrum of 5, the glucosyl $\mathrm{CH}_{2}\left(6^{\prime}\right)\left(\delta_{\mathrm{H}} 4.60\right.$, $4.22)$ correlated with the oleuropeoyl carboxylic C-atom C (7") ( $\left.\delta_{\mathrm{C}} 168.2\right)$, and the glucosyl H-C (1') $\left(\delta_{\mathrm{H}} 4.88\right)$ with and $\mathrm{C}(1)\left(\delta_{\mathrm{C}} 152.1\right)$ of $1,4-O$-disubstituted benzene ring moiety. The full assignments of all protons and carbons were preformed through the correlations in 2D-NMR spectra $\left({ }^{1} \mathrm{H}-{ }^{1} \mathrm{H}\right.$ COSY, HMQC and HMBC) of 5. All the data of ${ }^{1} \mathrm{H}-,{ }^{13} \mathrm{C}$-, and HMBC-NMR of compound 5 see Table 1, and key correlations and the structure of compound 5 see Figure 2. Methanolysis of 5 with $\mathrm{MeONa}$ in $\mathrm{MeOH}$ afforded 5a which was identified as $(R)$-oleuropeic acid methyl ester by comparing $[\alpha]_{\mathrm{D}}^{25}$ and ${ }^{1} \mathrm{H}$ - and ${ }^{13} \mathrm{C}$-NMR spectra data with the reported [4,5]. Based on the above evidence, the structure of 5 was determined to be $(R)$-4-hydroxylphenol $O$-(6- $O$-oleuropeoyl)- $\beta$-D-glucopyranoside.

Table 1. ${ }^{1} \mathrm{H}-\mathrm{NMR}$ (methanol- $d_{4}, 400 \mathrm{MHz}$ ), ${ }^{13} \mathrm{C}-\mathrm{NMR}$ (methanol- $d_{4}, 100 \mathrm{MHz}$ ) and HMBC data of compound 5 and $\mathbf{6}$ (TMS as the internal standard, $\delta$ in ppm $J$ in $\mathrm{Hz}$ ).

\begin{tabular}{|c|c|c|c|c|c|c|}
\hline \multirow{2}{*}{ No. } & \multicolumn{3}{|c|}{5} & \multicolumn{3}{|c|}{6} \\
\hline & $\boldsymbol{\delta}_{\mathbf{C}}$ & $\delta_{\mathrm{H}} J(\mathrm{~Hz})$ & HMBC $(\mathrm{H} \rightarrow \mathrm{C})$ & $\boldsymbol{\delta}_{\mathbf{C}}$ & $\delta_{\mathrm{H}} J(\mathbf{H z})$ & HMBC $(\mathrm{H} \rightarrow \mathrm{C})$ \\
\hline 1 & 152.1 & & & 153.0 & & \\
\hline 2 & 119.5 & $7.03 \mathrm{~d}(8.7)$ & $154.3,119.5$ & 104.5 & 6.80 br. s & $143.4,110.4$ \\
\hline 3 & 117.4 & $6.83 \mathrm{~d}(8.7)$ & $152.1,117.4$ & 149.2 & & \\
\hline $3-\mathrm{OCH}_{3}$ & & & & 56.8 & $3.90 \mathrm{~s}$ & 149.2 \\
\hline 4 & 154.3 & & & 143.4 & & \\
\hline 5 & 117.4 & $6.83 \mathrm{~d}(8.7)$ & $152.1,117.4$ & 116.2 & $6.75 \mathrm{~d}(8.2)$ & $153.0,149.2$ \\
\hline 6 & 119.5 & $7.03 \mathrm{~d}(8.7)$ & $119.5,154.3$ & 110.4 & $6.65 \mathrm{~d}(8.2)$ & $104.5,143.4$ \\
\hline $1^{\prime}$ & 103.3 & $4.88 \mathrm{~d}(7.5)$ & 168.2 & 103.6 & $4.83 \mathrm{~d}(7.8)$ & 153.0 \\
\hline $2^{\prime}$ & 75.2 & $3.29 \mathrm{~m}$ & & 75.2 & $3.51 \mathrm{~m}$ & \\
\hline $3^{\prime}$ & 78.4 & $3.46 \mathrm{~m}$ & & 78.2 & $3.53 \mathrm{~m}$ & \\
\hline $4^{\prime}$ & 72.2 & $3.36 \mathrm{~m}$ & & 72.3 & $3.43 \mathrm{~m}$ & \\
\hline $5^{\prime}$ & 75.6 & $3.75 \mathrm{~m}$ & & 75.8 & $3.71 \mathrm{~m}$ & \\
\hline $6^{\prime} \alpha$ & 65.6 & $4.60 \mathrm{~d}(11.5)$ & 168.2 & 65.2 & $4.59 \mathrm{~d}(11.5)$ & 168.2 \\
\hline $6^{\prime} \beta$ & & $4.22 \mathrm{dd}(11.5,7.7)$ & 168.2 & & $4.32 \mathrm{dd}(11.5,7.3)$ & 168.2 \\
\hline $1^{\prime \prime}$ & 131.5 & & & 131.5 & & \\
\hline $2^{\prime \prime}$ & 142.2 & 7.04 br. s & $168.2,29.1,45.7$ & 141.8 & 7.09 br. s & $168.2,45.4$ \\
\hline $3^{\prime \prime} \alpha$ & 29.1 & $2.48 \mathrm{~d}(16.5)$ & & 28.9 & $2.42 \mathrm{~d}(17.5)$ & \\
\hline $3^{\prime \prime} \beta$ & & $2.20 \mathrm{~m}$ & & & $2.12 \mathrm{~m}$ & \\
\hline $4 "$ & 45.7 & $1.63 \mathrm{~m}$ & & 45.4 & $1.65 \mathrm{~m}$ & \\
\hline $5^{\prime \prime} \alpha$ & 24.9 & $2.16 \mathrm{~m}$ & & 24.8 & $2.12 \mathrm{~m}$ & \\
\hline $5^{\prime \prime} \beta$ & & $1.32 \mathrm{~m}$ & & & $1.32 \mathrm{~m}$ & \\
\hline $6^{\prime \prime} \alpha$ & 26.9 & $2.59 \mathrm{~d}(16.0)$ & & 26.7 & $2.51 \mathrm{~d}(16.4)$ & \\
\hline $6^{\prime \prime} \beta$ & & $2.26 \mathrm{~m}$ & & & $2.21 \mathrm{~m}$ & \\
\hline $7^{\prime \prime}$ & 168.2 & & & 169.0 & & \\
\hline $8^{\prime \prime}$ & 72.2 & & & 73.2 & & \\
\hline $9^{\prime \prime}$ & 27.1 & $1.28 \mathrm{~s}$ & $45.7,72.2,26.6$ & 27.3 & $1.38 \mathrm{~s}$ & $45.4,73.2,26.8$ \\
\hline $10^{\prime \prime}$ & 26.6 & $1.28 \mathrm{~s}$ & $45.7,72.2,27.1$ & 26.8 & $1.28 \mathrm{~s}$ & $45.4,73.2,27.3$ \\
\hline
\end{tabular}

Note: The assignments were based on DEPT, HMQC, ${ }^{1} \mathrm{H}-{ }^{1} \mathrm{H}$ COSY, and HMBC experiments. 


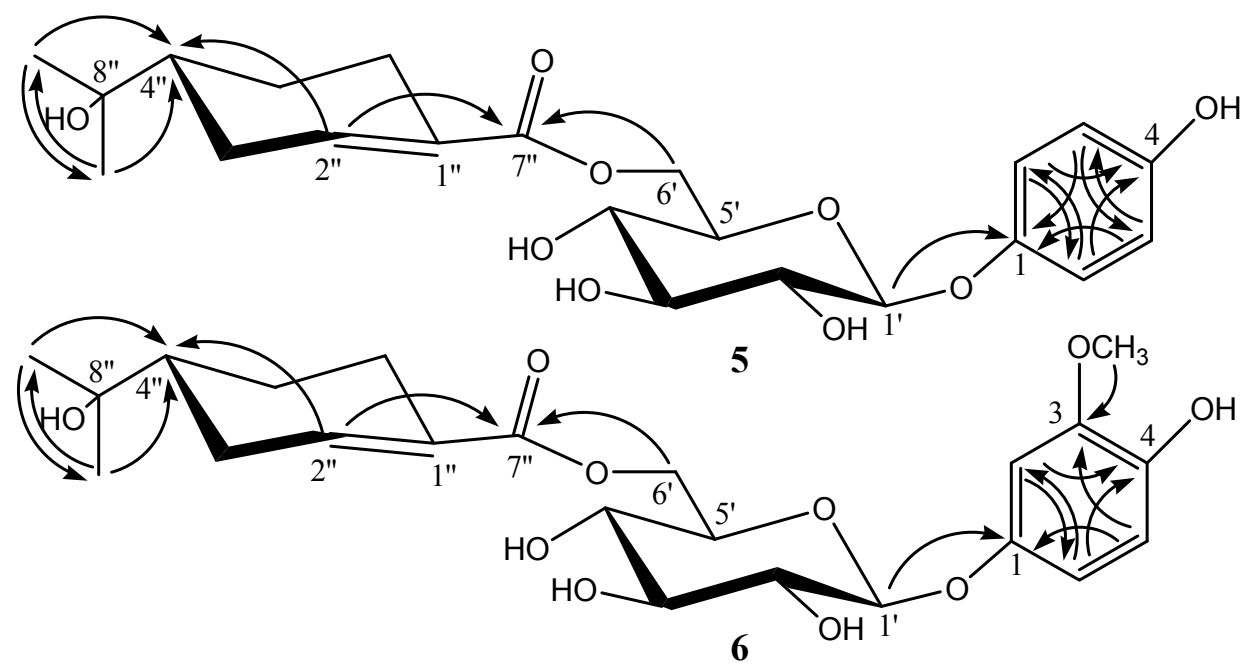

Figure 2. The Key HMBC Correlation of compound $\mathbf{5}$ and $\mathbf{6}$ (arrows point from proton to carbon).

Compound 6 was obtained as colorless needles. Its molecular formula $\mathrm{C}_{23} \mathrm{H}_{32} \mathrm{O}_{10}$ was elucidated from the HRESIMS $m / z$ 469.2086 [M $+\mathrm{H}]^{+}$(calcd for $\mathrm{C}_{23} \mathrm{H}_{33} \mathrm{O}_{10}, 469.2074$ ). Acidic hydrolysis of 6 also yielded D-glucose as a sugar residue. The ${ }^{1} \mathrm{H}$ - and ${ }^{13} \mathrm{C}$-NMR spectra of $\mathbf{6}$ were similar to those of $\mathbf{5}$, except for the signals of benzene ring moiety. The ${ }^{1} \mathrm{H}$ - and ${ }^{13} \mathrm{C}-\mathrm{NMR}$ spectra showed a typical three proton $\mathrm{ABX}$ aromatic spin system at $\delta_{\mathrm{H}} 6.75(1 \mathrm{H}, \mathrm{d}, J=8.2 \mathrm{~Hz}), 6.65(1 \mathrm{H}, \mathrm{d}, J=8.2 \mathrm{~Hz}), 6.80(1 \mathrm{H}$, br. s) and six aromatic C-atom signals at $\delta_{\mathrm{C}} 153.0(\mathrm{C}), 149.2(\mathrm{C}), 143.4(\mathrm{C}), 116.2(\mathrm{CH}), 110.4(\mathrm{CH})$ and $104.5(\mathrm{CH})$, as well as one signal from a $\mathrm{MeO}$ group $\left.\left[\delta_{\mathrm{H}} 3.90(\mathrm{~s}, 3 \mathrm{H}), \delta_{\mathrm{C}} 56.8\right)\right]$, suggesting that there is a 1,3,4-O-trisubstituted benzene ring moiety in compound $\mathbf{6}$ in stead of a 1,4-O-disubstituted benzene ring moiety, and one additional methoxy group. The positions of the oleuropeoyl ester, glycosidic linkages and $\mathrm{MeO}$ group in $\mathbf{6}$ were also established by 2D-NMR experiments. In the HMBC spectrum of $\mathbf{6}$, the glucosyl $\mathrm{CH}_{2}\left(6^{\prime}\right)\left(\delta_{\mathrm{H}} 4.59,4.32\right)$ correlated with the oleuropeoyl carboxylic $\mathrm{C}$-atom $\mathrm{C}\left(7^{\prime \prime}\right)\left(\delta_{\mathrm{C}} 169.0\right)$, the glucosyl $\mathrm{H}-\mathrm{C}\left(1^{\prime}\right)\left(\delta_{\mathrm{H}} 4.83\right)$ with $\mathrm{C}(1)\left(\delta_{\mathrm{C}} 153.0\right)$ of the 1,3,4-O-trisubstituted benzene ring moiety, and the $\mathrm{MeO}$ group $\delta_{\mathrm{H}} 3.90$ correlated with $\mathrm{C}(3)\left(\delta_{\mathrm{c}} 149.2\right)$ of 1,3,4-O-trisubstituted benzene ring moiety. The full assignments of all protons and carbons were preformed through the correlations in 2D-NMR spectra $\left({ }^{1} \mathrm{H}-{ }^{1} \mathrm{H}\right.$ COSY, HMQC and HMBC) of 6. All the data of ${ }^{1} \mathrm{H}-,{ }^{13} \mathrm{C}-$, and HMBC-NMR of compound 6 see Table 1, and key correlations and the structure of compound 6 see Figure 2. Methanolysis of $\mathbf{6}$ also afforded 5a. Thus, the structure of $\mathbf{6}$ was determined to be $(R)-3$-methoxy-4hydroxylphenol $O$-(6-O-oleuropeoyl)- $\beta$-D-glucopyranoside.

Using similar methods as described above, compounds 1-4 and $\mathbf{7}$ were identified as (-)-Epicatechin (1) [6], 5,7,4'-trihydroxy-flavonoid-8-C- $\beta$-D-glucopyranoside (2) [7], 1-(4-hydroxy-3-methoxy-phenyl)2-[4-(3- $\alpha$-L-rhamnopyranoxypropyl)-2-methoxyphenoxy]-1,3-propane-diol (erythro) (3) [8], 1-(4-hydroxy3-methoxyphenyl)-2-[4-(3- $\alpha$-L-rhamnopyranoxypropyl)-2-methoxyphenoxy]-1,3-propanediol (threo) (4) [8], quercetin-3-O-rutinoside (7) [9].

Compounds 1-7 were next assayed for their antioxidant activity with DPPH free radicals, and the results are shown in Table 2 . The data proved that (-)-Epicatechin showed strongest antioxidant activity. 
Table 2. IC50 values of compounds 1-7.

\begin{tabular}{cc}
\hline Compound & $\mathbf{I C}_{\mathbf{5 0}}\left(\boldsymbol{\mu} \mathbf{g} \cdot \mathbf{m L}^{-\mathbf{1}}\right)$ \\
\hline $\mathbf{1}$ & $9.85 \pm 0.5$ \\
$\mathbf{2}$ & $357.1 \pm 6.2$ \\
$\mathbf{3}$ & $520.9 \pm 7.6$ \\
$\mathbf{4}$ & $522.3 \pm 8.1$ \\
$\mathbf{5}$ & $610.8 \pm 6.1$ \\
$\mathbf{6}$ & $620.1 \pm 7.3$ \\
$\mathbf{7}$ & $37.5 \pm 0.3$ \\
\hline
\end{tabular}

Note: All values are averages of at least three runs in Table 2.

\section{Experimental Section}

\subsection{General Information}

NMR spectra were recorded on a Bruker AV-400 spectrometer (Bruke Corporation, Faellanden, Switzerland). UV Spectra were recorded on a Shimadzu UV-2401A spectrometer (Shimadzu Corporation, Kyoto, Japan). HR-ESI-MS were recorded on a Bruker microOTOF-Q II mass spectrometer (Bruke Corporation, Bremen, Germany). Optical rotations were measured with a HORIBA SEPA-300 high-sensitive polarimeter (Horiba Ltd, Kyoto, Japan). Melting points (m.p.) were measured with a X-4 Microscopic melting point apparatus (Shanghai Hui Tong Optical Instrument Co., Ltd, Shanghai, China). HPLC was performed Shimadzu LC-10A with a SPD-10A detector (Shimadzu Corporation, Kyoto, Japan) and Gemini $5 \mu \mathrm{C} 18110 \mathrm{~A}$ column $(250 \mathrm{~mm} \times 10.00 \mathrm{~mm}, 5 \mu \mathrm{m}$, flow rate: $3.0 \mathrm{~mL} / \mathrm{min}$, Phenomenex, Torrance, CA, USA). GC was performed an Agilent 7820A gas chromatograph with a quartz capillary column $(30 \mathrm{~mm} \times 0.32 \mathrm{~mm} \times 0.25 \mu \mathrm{m}$, Agilent Technolgies Inc., Santa Clara, CA, USA); detection, FID. Column chromatography was performed on silica gel (200-300 mesh, Qingdao Marine Chemical Inc., Qingdao, China), D101 polyporous resin (Tianjin Pesticide Co., LTD., Resin Branch, Tianjin, China), polyamide (80-120 mesh, Taizhou Luqiao Sijia Biochemical Plastic Factory, Taizhou, China) and MCI-gel CHP-20P (75-150 $\mu \mathrm{m}$; Mitsubishi Chemical Co., Tokyo, Japan). TLC was performed on glass precoated silica gel $\mathrm{GF}_{254}$ plates (Qingdao Haiyang Chemical Co., Ltd, Qingdao, China), detection under UV light or by spraying with $10 \% \mathrm{H}_{2} \mathrm{SO}_{4}$ in $95 \%$ EtOH followed by heating. The bioactivities were measured on a DNM-9602 Enzyme immunoassay spectrophotometer (Beijing, China), using 1,1-diphenyl-2-picrylhydrazyl free radical (DPPH) (Sigma-Aldrich, Shanghai, China). The fruits of Viburnum sargentii Koehne were collected in Changchun District of Jilin Province, China. They were identified by Jing-min Zhang of the School of Pharmaceutical Sciences, Jilin University.

\subsection{Extraction and Isolation}

The fresh fruits of $V$. sargentii $(8 \mathrm{~kg}$ ) were extracted with $70 \%$ aqueous ethanol at room temperature $(3 \mathrm{~L} \times 10 \mathrm{~L}$, weekly). The extracts were concentrated under reduced pressure and then subjected to D101 polyporous resin column chromatography eluated with $\mathrm{H}_{2} \mathrm{O}, 10 \%$ aqueous ethanol, 30\% aqueous ethanol, $60 \%$ aqueous ethanol and $95 \%$ aqueous ethanol. The eluate of $30 \%$ ethanol was chromatographed over silica gel, eluting with $\mathrm{CHCl}_{3}-\mathrm{EtOAc}-\mathrm{MeOH}-\mathrm{H}_{2} \mathrm{O}$ (3.5:1.2:4:1.2, v/v, lower 
layer), to afford four fractions, Frs. 1-4. Compound 1 (521 mg) was recrystallized from Fr. 1, Fr. 2 and 4 were further chromatographicly separated by gradient elution with $\mathrm{MeOH}-\mathrm{H}_{2} \mathrm{O}$ (from $0 \%$ to $65 \%$, $5 \%$ a time, $v / v)$, and recrystallization of compound $2(45 \mathrm{mg}$ ) were obtained from Fr. 2 and compound 7 (100 mg) from Fr. 4. Fr. 3 was further subjected to silica gel column chromatography eluting with $\mathrm{CHCl}_{3}-\mathrm{EtOAc}-\mathrm{MeOH}-\mathrm{H}_{2} \mathrm{O}$ (3.0:1.2:4:1.2, v/v, lower layer) to afford three subfractions, Frs. 3a-c. They were further isolated by semi-preparative RP-HPLC using acetonitrile- $\mathrm{H}_{2} \mathrm{O}$ as the mobile phase. Compound 3 (47 mg) and 4 (101 mg) were obtained from Fr. 3a with gradient elution (10\%-15\% acetonitrile from $0.00-10.00 \mathrm{~min}, 15 \%-18 \%$ acetonitrile from 10.00-20.0 $0 \mathrm{~min}, 18 \%$ acetonitrile from 20.00-50.00 $\mathrm{min}$ ), and by using 18\% acetonitrile as the mobile phase compound 5 (62 $\mathrm{mg}$ ) from Fr. $3 \mathrm{~b}$ and compound 6 (57 mg) from Fr. $3 \mathrm{c}$.

Compound 1: Pale amorphous powder, yielded a positive reaction to $\mathrm{FeCl}_{3}$ reagent. $\mathrm{mp} 234-236{ }^{\circ} \mathrm{C}$. $[\alpha]_{\mathrm{D}}^{25}-56.8\left(c\right.$ 1.0, MeOH). UV (MeOH), $\lambda \max 217,280 \mathrm{~nm}$. HRESIMS $m / z 291.0877[\mathrm{M}+\mathrm{H}]^{+}$(calcd for $\left.\mathrm{C}_{15} \mathrm{H}_{15} \mathrm{O}_{6}, 291.0869\right)$. ${ }^{1} \mathrm{H}-\mathrm{NMR}$ (DMSO- $\left.d_{6}, 400 \mathrm{MHz}\right) \delta: 2.48(1 \mathrm{H}, \mathrm{dd}, J=16.0,3.6 \mathrm{~Hz}, \mathrm{H}-4 \mathrm{a})$, $2.68(1 \mathrm{H}, \mathrm{dd}, J=16.0,4.8 \mathrm{~Hz}, \mathrm{H}-4 \mathrm{~b}), 4.01(1 \mathrm{H}, \mathrm{m}, \mathrm{H}-3), 4.65(1 \mathrm{H}, \mathrm{d}, J=4.8 \mathrm{~Hz}, \mathrm{H}-2), 5.89(1 \mathrm{H}, \mathrm{d}$, $J=2.0 \mathrm{~Hz}, \mathrm{H}-8), 5.72(1 \mathrm{H}, \mathrm{d}, J=2.0 \mathrm{~Hz}, \mathrm{H}-6), 6.64\left(1 \mathrm{H}, \mathrm{dd}, J=8.0,1.6 \mathrm{~Hz}, \mathrm{H}-66^{\prime}\right), 6.67(1 \mathrm{H}, \mathrm{d}$, $\left.J=8.0 \mathrm{~Hz}, \mathrm{H}-5^{\prime}\right), 6.89\left(1 \mathrm{H}, \mathrm{d}, J=1.6 \mathrm{~Hz}, \mathrm{H}-2^{\prime}\right) .{ }^{13} \mathrm{C}-\mathrm{NMR}$ (DMSO-d6,100 MHz) $\delta: 78.0(\mathrm{C}-2), 64.9$ (C-3), 28.2 (C-4), 156.5 (C-5), 94.1 (C-6), 156.2 (C-7), 95.1 (C-8), 155.8 (C-9), 98.5 (C-10), 130.6 $\left(\mathrm{C}-1^{\prime}\right), 114.9\left(\mathrm{C}-2^{\prime}\right), 144.5\left(\mathrm{C}-3^{\prime}\right), 144.4\left(\mathrm{C}-4^{\prime}\right), 114.7$ (C-5'), $117.9\left(\mathrm{C}-6^{\prime}\right)$.

Compound 2: Yellow amorphous powder, yielded a positive reaction to $\mathrm{FeCl}_{3}$ reagent. $\mathrm{mp} 238-240{ }^{\circ} \mathrm{C}$. UV (MeOH), $\lambda_{\max } 268,339$. HRESIMS $m / z 433.1126[\mathrm{M}+\mathrm{H}]^{+}$(calcd for $\mathrm{C}_{21} \mathrm{H}_{21} \mathrm{O}_{10}, 433.1135$ ). ${ }^{1} \mathrm{H}-\mathrm{NMR}$ $\left(\mathrm{DMSO}-d_{6}, 400 \mathrm{MHz}\right) \delta: 8.02\left(2 \mathrm{H}, \mathrm{d}, J=8.0 \mathrm{~Hz}, \mathrm{H}-2^{\prime}, 6^{\prime}\right), 6.89\left(2 \mathrm{H}, \mathrm{d}, J=8.0 \mathrm{~Hz}, \mathrm{H}-3^{\prime}, 5^{\prime}\right), 6.77(1 \mathrm{H}$, s, H-3), $6.28(1 \mathrm{H}, \mathrm{s}, \mathrm{H}-6), 4.69\left(1 \mathrm{H}, \mathrm{d}, J=8.0 \mathrm{~Hz}\right.$, Glu-H-1). ${ }^{13} \mathrm{C}-\mathrm{NMR}$ (DMSO-d6, $\left.100 \mathrm{MHz}\right) \delta$ : (C-7), 104.6 (C-8), 156.0 (C-9), 104.0 (C-10), 121.6 (C-1'), 128.9 (C-2'), 115.8 (C-3'), 160.3 (C-4'), 115.8 (C-5'), 128.9 (C-6'), 73.3 (Glu-1), 70.8 (Glu-2), 78.6 (Glu-3), 70.5 (Glu-4), 81.8 (Glu-5), 61.3 (Glu-6).

Compound 3: Pale amorphous powder, yielded a positive reaction to $\mathrm{FeCl}_{3}$ reagent. mp $190-192{ }^{\circ} \mathrm{C}$. $[\alpha]_{\mathrm{D}}^{25}-26.9(c 0.30, \mathrm{MeOH}) . \mathrm{UV}(\mathrm{MeOH}), \lambda_{\max } 228,280$. HRESIMS $m / z 525.2328[\mathrm{M}+\mathrm{H}]^{+}$(calcd for $\left.\mathrm{C}_{26} \mathrm{H}_{37} \mathrm{O}_{11}, 525.2336\right) .{ }^{1} \mathrm{H}-\mathrm{NMR}$ (methanol- $\left.d_{4}, 400 \mathrm{MHz}\right) \delta: 7.10\left(1 \mathrm{H}, \mathrm{s}, \mathrm{H}-2^{\prime}\right), 6.92(2 \mathrm{H}, \mathrm{d}, J=8.0 \mathrm{~Hz}$, H-6',5), 6.88 (1H, s, H-2), $6.83\left(1 \mathrm{H}, \mathrm{d}, J=8.0 \mathrm{~Hz}, \mathrm{H}-5^{\prime}\right), 6.75(1 \mathrm{H}, \mathrm{d}, J=8.0 \mathrm{~Hz}, \mathrm{H}-6), 4.92(1 \mathrm{H}, \mathrm{d}$, $\left.J=7.6 \mathrm{~Hz}, \mathrm{H}-7^{\prime}\right), 4.73$ (1H, s, Rha-H-1), 4.38 (1H, m, H-8'), 3.94 (1H, m, H-9'a), 3.83 (1H, m, H-9'b), 3.74 (1H, m, H-9a), 3.46 (1H, m, H-9b), 2.72 (2H, m, H-7), 1.96 (2H, m, H-8), 3.90 (3H, s, 3'-OCH3), $3.88\left(3 \mathrm{H}, \mathrm{s}, 3-\mathrm{OCH}_{3}\right), 1.32\left(3 \mathrm{H}, \mathrm{d}, J=6.4 \mathrm{~Hz}\right.$, Rha-H-6). ${ }^{13} \mathrm{C}-\mathrm{NMR}$ (methanol- $\left.d_{4}, 100 \mathrm{MHz}\right) \delta: 138.1$ (C-1), 114.3 (C-2), 152.2 (C-3), 147.5 (C-4), 119.9 (C-5), 122.2 (C-6), 33.3 (C-7), 32.7 (C-8), 68.0 (C-9), $134.4\left(\mathrm{C}-1^{\prime}\right), 112.1\left(\mathrm{C}-2^{\prime}\right), 149.0$ (C-3'), 147.3 (C-4'), 116.0 (C-5'), 121.2 (C-6'), 74.4 (C-7'), 86.9 (C-8'), 62.4 (C-9'), 56.8 (3'-OCH3), 56.7 (3'-OCH3), 102.0 (Rha-1), 72.8 (Rha-2), 72.6 (Rha-3), 74.3 (Rha-4), 70.1 (Rha-5), 18.3 (Rha-6).

Compound 4: Pale amorphous powder, yielded a positive reaction to $\mathrm{FeCl}_{3}$ reagent. mp $186-188{ }^{\circ} \mathrm{C}$. $[\alpha]_{\mathrm{D}}^{25}-29.5(c 0.30, \mathrm{MeOH})$. UV (MeOH), $\lambda \max 230,280$. HRESIMS $m / z 525.2325[\mathrm{M}+\mathrm{H}]^{+}$(calcd for $\left.\mathrm{C}_{26} \mathrm{H}_{37} \mathrm{O}_{11}, 525.2336\right) .{ }^{1} \mathrm{H}-\mathrm{NMR}$ (methanol- $\left.d_{4}, 400 \mathrm{MHz}\right) \delta: 7.11\left(1 \mathrm{H}, \mathrm{s}, \mathrm{H}-2^{\prime}\right), 7.07(\mathrm{H}, \mathrm{d}, J=8.0 \mathrm{~Hz}$, H-5), 6.95 (1H, d, $\left.J=8.0 \mathrm{~Hz}, \mathrm{H}-6^{\prime}\right), 6.94(1 \mathrm{H}, \mathrm{s}, \mathrm{H}-2), 6.85$ (1H, d, $\left.J=8.0 \mathrm{~Hz}, \mathrm{H}-5^{\prime}\right), 6.80$ (1H, d, 
$J=8.0 \mathrm{~Hz}, \mathrm{H}-6), 4.96\left(1 \mathrm{H}, \mathrm{d}, J=6.8 \mathrm{~Hz}, \mathrm{H}-7^{\prime}\right), 4.73(1 \mathrm{H}, \mathrm{s}, \mathrm{Rha}-\mathrm{H}-1), 4.30\left(1 \mathrm{H}, \mathrm{m}, \mathrm{H}-8^{\prime}\right), 3.80(1 \mathrm{H}$, m, H-9'a), 3.74 (1H, m, H-9'b), 3.54 (1H, m, H-9a), 3.45 (1H, m, H-9b), 2.74 (2H, m, H-7), 1.97 (2H, m, H-8), $3.95\left(3 \mathrm{H}, \mathrm{s}, 3{ }^{\prime}-\mathrm{OCH}_{3}\right), 3.91\left(3 \mathrm{H}, \mathrm{s}, 3-\mathrm{OCH}_{3}\right), 1.32\left(3 \mathrm{H}, \mathrm{d}, J=6.0 \mathrm{~Hz}\right.$, Rha-H-6). ${ }^{13} \mathrm{C}-\mathrm{NMR}$ (methanol-d4, $100 \mathrm{MHz}$ ) $\delta: 138.2$ (C-1), 114.2 (C-2), 152.0 (C-3), 148.0 (C-4), 119.9 (C-5), 122.4 (C-6), 33.3 (C-7), 32.7 (C-8), 68.0 (C-9), 134.1 (C-1'), 112.0 (C-2'), 149.1 (C-3'), 147.5 (C-4'), 116.2 (C-5'), $121.1\left(\mathrm{C}-6^{\prime}\right), 74.5\left(\mathrm{C}-7^{\prime}\right), 88.0\left(\mathrm{C}-8^{\prime}\right), 62.2\left(\mathrm{C}-9^{\prime}\right), 56.9\left(3^{\prime}-\mathrm{OCH}_{3}\right), 56.7$ (3'-OCH $), 102.0$ (Rha-1), 72.8 (Rha-2), 72.7 (Rha-3), 74.3 (Rha-4), 70.1 (Rha-5), 18.3 (Rha-6).

Compound 5: colorless needles $(\mathrm{MeOH})$, yielded a positive reaction to $\mathrm{FeCl}_{3}$ reagent. mp $198-200{ }^{\circ} \mathrm{C}$, $[\alpha]_{\mathrm{D}}^{25}-17.5(c 1.0, \mathrm{MeOH})$. UV (MeOH), $\lambda_{\max } 210,261 \mathrm{~nm}$. HRESIMS $m / z 439.1957[\mathrm{M}+\mathrm{H}]^{+}(\mathrm{calcd}$ for $\mathrm{C}_{22} \mathrm{H}_{31} \mathrm{O} 9$, 439.1968). ${ }^{1} \mathrm{H}-\mathrm{NMR}$ (methanol- $d_{4}, 400 \mathrm{MHz}$ ), see Table 1 ; ${ }^{13} \mathrm{C}-\mathrm{NMR}$ (methanol- $d_{4}$, $100 \mathrm{MHz})$, see Table 1.

Compound 6: colorless needles $(\mathrm{MeOH})$, yielded a positive reaction to $\mathrm{FeCl}_{3}$ reagent. mp $190-192{ }^{\circ} \mathrm{C}$, $[\alpha]_{\mathrm{D}}^{25}-24.5(c \text { 0.8, MeOH). UV (MeOH), } \lambda \max 215,269 \mathrm{~nm} \text {. HRESIMS } m / z \text { 469.2086 [M + H] }]^{+}$(calcd for $\mathrm{C}_{23} \mathrm{H}_{33} \mathrm{O}_{10}, 469.2074$ ). ${ }^{1} \mathrm{H}-\mathrm{NMR}$ (methanol- $d_{4}, 400 \mathrm{MHz}$ ), see Table 1 ; ${ }^{13} \mathrm{C}-\mathrm{NMR}$ (methanol- $d_{4}$, $100 \mathrm{MHz})$, see Table 1.

Compound 7: Yellow amorphous powder, yielded a positive reaction to $\mathrm{FeCl}_{3}$ reagent. mp $185-187{ }^{\circ} \mathrm{C}$. UV $(\mathrm{MeOH}), \lambda_{\max } 259,359$. HRESIMS $m / z 611.1620[\mathrm{M}+\mathrm{H}]^{+}$(calcd for $\mathrm{C}_{27} \mathrm{H}_{31} \mathrm{O}_{16}, 611.1612$ ). ${ }^{1} \mathrm{H}-\mathrm{NMR}\left(\mathrm{DMSO}-d_{6}, 400 \mathrm{MHz}\right) \delta: 6.19(1 \mathrm{H}, \mathrm{s}, \mathrm{H}-6), 6.38(1 \mathrm{H}, \mathrm{s}, \mathrm{H}-8), 7.53\left(1 \mathrm{H}, \mathrm{d}, J=1.6 \mathrm{~Hz}, \mathrm{H}-2^{\prime}\right)$, $6.84\left(1 \mathrm{H}, \mathrm{d}, J=8.0 \mathrm{~Hz}, \mathrm{H}-5^{\prime}\right), 7.54\left(1 \mathrm{H}, \mathrm{dd}, J=8.0,1.6 \mathrm{~Hz}, \mathrm{H}-6^{\prime}\right), 5.34$ (1H, d, $\left.J=6.8 \mathrm{~Hz}, \mathrm{Glc}-\mathrm{H}-1\right)$, 4.39 (1H, s, Rha-H-1), 1.00 (3H, d, $J=6.0 \mathrm{~Hz}$, Rha-H-6). ${ }^{13} \mathrm{C}-\mathrm{NMR}$ (DMSO-d6, $\left.100 \mathrm{MHz}\right) \delta: 156.4(\mathrm{C}-2)$, 133.3 (C-3), 177.3 (C-4), 161.2 (C-5), 98.6 (C-6), 164.1 (C-7), 93.5 (C-8), 156.5 (C-9), 103.9 (C-10), $121.1\left(\mathrm{C}-1^{\prime}\right), 115.2$ (C-2'), 144.7 (C-3'), 148.4 (C-4'), 116.2 (C-5'), 121.5 (C-6'), 101.2 (Glc-1), 74.0 (Glc-2), 76.4 (Glc-3), 70.0 (Glc-4), 75.9 (Glc-5), 66.9 (Glc-6), 100.7 (Rha-1), 70.5 (Rha-2), 70.3 (Rha-3), 71.8 (Rha-4), 68.2 (Rha-5), 17.7 (Rha-6).

\subsection{Acid Hydrolysis of $\mathbf{5}$ and $\mathbf{6}$}

Compounds 5 and 6 (each $6 \mathrm{mg}$ ) were hydrolyzed with $1.5 \mathrm{~N} \mathrm{HCl}(2 \mathrm{~mL})$ at $80{ }^{\circ} \mathrm{C}$ for $5 \mathrm{~h}$. The mixture was then neutralized with $\mathrm{NaOH}(1 \mathrm{~N})$. The mixture was passed through MCI-gel CHP-20P, developing with $\mathrm{H}_{2} \mathrm{O}$. The $\mathrm{H}_{2} \mathrm{O}$ eluate was evaporated to dryness. The dry powders were dissolved in pyridine $(2 \mathrm{~mL})$, L-cysteine methyl ester hydrochloride $(1.5 \mathrm{mg}$ ) was added, and the mixture was heated at $60{ }^{\circ} \mathrm{C}$ for $1 \mathrm{~h}$. Trimethylsilylimidazole $(1.5 \mathrm{~mL})$ was added, and the mixture was heated at $60{ }^{\circ} \mathrm{C}$ for another $0.5 \mathrm{~h}$. An aliquot $(4 \mu \mathrm{L})$ of the supernatant was subjected to $\mathrm{GC}$ analysis under the following conditions: column temp $180-280{ }^{\circ} \mathrm{C}$ at $3 \mathrm{deg} / \mathrm{min}$, carrier gas $\mathrm{N}_{2}(1 \mathrm{~mL} / \mathrm{min})$, injector and detector temp $250{ }^{\circ} \mathrm{C}$, split ratio 1:50. The configurations of D-gluose for compounds $\mathbf{5}$ and $\mathbf{6}$ were determined by comparison of the retentions times of the corresponding derivatives with standard D-glucose (retention time: $19.208 \mathrm{~min}$ ), respectively. 


\subsection{Methanolysis of $\mathbf{5}$ and $\mathbf{6}$}

A solution of $5(12 \mathrm{mg})$ in $0.02 \mathrm{M} \mathrm{NaOMe}-\mathrm{MeOH}(2 \mathrm{~mL})$ was kept standing at room temperature for $12 \mathrm{~h}$. The solution was then subjected to MCI-gel CHP-20P column chromatography, eluting with $\mathrm{H}_{2} \mathrm{O}, 60 \%$ and $100 \% \mathrm{MeOH}$ to give $(R)$-methyl oleuropeic acid methyl ester (5a) (3.0 mg): colorless oil; $[\alpha]_{\mathrm{D}}^{25}+65.5\left(c 0.2, \mathrm{CHCl}_{3}\right) ;{ }^{1} \mathrm{H}-\mathrm{NMR}\left(\mathrm{CDCl}_{3}, 400 \mathrm{MHz}\right) \delta 7.06(1 \mathrm{H}, \mathrm{m}, \mathrm{H}-2), 2.30,2.00$ (m, H-3), 1.53 (m, H-4), 2.01, 1.22 (m, H-5), 2.51, 2.12 (m, H-6), 1.20 (3H, s, H-9), 1.21 (3H, s, H-10), 3.72 $\left(3 \mathrm{H}, \mathrm{s}, \mathrm{OCH}_{3}\right) ;{ }^{13} \mathrm{C}-\mathrm{NMR}\left(\mathrm{CDCl}_{3}, 100 \mathrm{MHz}\right) \delta 130.2$ (C-1), $139.9(\mathrm{C}-2), 27.4(\mathrm{C}-3), 44.5(\mathrm{C}-4), 23.4$ (C-5), 25.1 (C-6), 167.8 (C-7), 72.2 (C-8), 27.4 (C-9), 26.6 (C-10), $51.6\left(\mathrm{OCH}_{3}\right)$. Similar methanolysis of 6 also gave $\mathbf{5 a}\left([\alpha]_{\mathrm{D}}^{25}+62.7\left(c 0.18, \mathrm{CHCl}_{3}\right)\right)$.

\subsection{Bioactivity Assay}

The antioxidant activities of compounds 1-7 were assessed according to their DPPH (1,1-diphenyl2-picrylhydrazyl free radical, Sigma-Aldrich, Shanghai, China) scavenging ability. Reaction mixtures, containing $0.5 \mathrm{~mL}$ of the relevant compound (dissolved in EtOH) and $2.5 \mathrm{~mL}$ of a $100 \mu \mathrm{M} \mathrm{DPPH}$ ethanolic solution, were added to 96-well microtiter plates and incubated at $37{ }^{\circ} \mathrm{C}$ for $30 \mathrm{~min}$. Absorbances were measured at $515 \mathrm{~nm}$. Percent inhibition was determined by comparison with an EtOH-treated control group. $\mathrm{IC}_{50}$ values denote the concentration of samples required to scavenge $50 \%$ of the DPPH free radicals.

\section{Conclusions}

Compounds 5 and $\mathbf{6}$ are new monoterpene phenolic glycosides. Compounds 1, 3 and $\mathbf{4}$ were isolated from the Viburnum genus for the first time, and compounds $\mathbf{2}$ and $\mathbf{7}$ from the Viburnum sargentii Koehne for the first time. Compounds 1-7 were also assayed for their antioxidant activities with DPPH free radicals, and the data proved that (-)-Epicatechin showed the strongest antioxidant activity.

\section{Acknowledgments}

The authors gratefully thank Jing-Min Zhang (School of Pharmaceutical Sciences, Jilin University) for the identification of plants.

\section{Author Contributions}

Guang-Shu Wang designed research, analyzed the data and wrote the paper; Yang Xie, Jing Wang, Yan-Mei Geng, Zhi Zhang and Yan-Fei Qu performed research. All authors read and approved the final manuscript.

\section{Conflicts of Interest}

The authors declare no conflict of interest. 


\section{References}

1. The Editorial Board of Zhong Hua Ben Cao of State Administration of Traditional Chinese Medicine of the People's Republic of China. Zhong Hua Ben Cao, 1st ed.; Scientific and Technical Publishers: Shanghai, China, 1999; Volume 7, p. 559.

2. Li, Y.B.; Zhang, L.; Yan, J. Study on the cough effect of Viburnum sargentii Koehne fruit. Inf. Tradit. Chin. Med. 2002, 19, 60.

3. Zhang, C.X.; Chen, H.; Ren, Y.L.; Zhu, J. Study on the antibacterial effect of Viburnum sargentii Koehne fruit extract. J. Anhui Agric. Sci. 2010, 38, 11767-11768, 11782.

4. Manns, D.; Hartmann, R. Monoterpene glucosides from Cunila Spicata. Planta Med. 1994, 60, 467-469.

5. Ito, H.; Koreishi, M.; Tokuda, H.; Nishino, H.; Yoshida, T. Cypellocarpins A-C, phenol glycosides esterified with oleuropeic acid, from Eucalyptus Cypellocarpa. J. Nat. Prod. 2000, 63, $1253-1257$.

6. Wang, Q.H.; Hubisihalatu; Wu, J.S.; Rong, J.; Narenchaoketu; Dai, N.Y.T. Chemical constituents of Helianthemum ordosicum. Chin. Tradit. Herb. Drugs 2014, 45, 2607-2610.

7. Li, H.Y.; Sun, J.Y.; Dai, S.W. Stduies on chemical constituents from Phyllostachys pubescens. J. Chin. Med. Mater. 2003, 26, 563-564.

8. Miyase, T.; Ueno, A.; Takizawa, N.; Kobayashi, H.; Oguchi, H. Studies on the glycosides of Epimedium grandiflorum MORR. var. thunbergianum (MIQ.) NAKAI. II. Chem. Pharm. Bull. 1987, 35, 3713-3719.

9. Chen, L.S.; Liang, X.X.; Cai, Z.Y.; Li, L.H. Chemical constituents from Tamarix Chinensis. Chin. Tradit. Herb. Drug 2014, 45, 1829-1833.

Sample Availability: Samples of the compounds 1, 2, 5 and $\mathbf{7}$ are available from the authors.

(C) 2015 by the authors; licensee MDPI, Basel, Switzerland. This article is an open access article distributed under the terms and conditions of the Creative Commons Attribution license (http://creativecommons.org/licenses/by/4.0/). 